\title{
A Case of Unilateral Adrenal Hemorrhage in Pulmonary Tuberculosis Presenting as Acute Abdominal Pain
}

\author{
Girish N Nadkarni ${ }^{\mathrm{a}, \mathrm{g}}$,Vijay Kanakadandi ${ }^{\mathrm{b}}$, Manpreet S Sabharwal ${ }^{\mathrm{c}}$, Natraj Ammakkanavar ${ }^{\mathrm{d}}$, \\ Shiv Kumar Agarwale ${ }^{\mathrm{e}}$, Hari Priya Sanjani ${ }^{\mathrm{e}}$, Priya Simoes ${ }^{\mathrm{e}}$, Narender Annapureddy ${ }^{\mathrm{f}}$
}

\begin{abstract}
A 75-year-old male presented with productive cough; unintentional weight loss and constitutional symptoms since 1 month and was diagnosed with pulmonary tuberculosis. He was then started on appropriate antitubercular therapy. During his hospitalization, he developed acute abdominal pain. Laboratory testing was consistent with acute adrenal insufficiency and was diagnosed to be acute adrenal insufficiency secondary to unilateral hemorrhage on a computerized tomography scan. He was treated with corticosteroids and had recovery of adrenal function. Through this case, we wish to illustrate the importance of recognizing early this rare but potentially life threatening complication of pulmonary tuberculosis.
\end{abstract}

Keywords: Tuberculosis; Adrenal hemorrhage; Adrenal insufficiency; Corticosteroids

\section{Introduction}

Adrenal hemorrhage may have a nonspecific presentation

\footnotetext{
Manuscript accepted for publication August 13, 2012

${ }^{a}$ Department of Nephrology, The Mount Sinai School Of Medicine, One Gustave L. Levy Place, No:1243, New York, USA

${ }^{\mathrm{b}}$ Department of Gastroenterology, Kansas City VA Medical

Center,4801 E Linwood Blvd, Kansas City, USA

${ }^{\mathrm{c}}$ Department of Cardiology, Albert Einstein College Of Medicine 1300

Morris Park Avenue, No: 10461, Bronx, NY, USA

${ }^{\mathrm{d}}$ Department Of Hematology/Oncology, Indiana University, Joseph E.

Walther Hall, 980 West Walnut Street R3 C312, Indianapolis, Indiana,

USA

eDepartment of Medicine, St. Luke's-Roosevelt Hospital Center, Columbia University College of Physicians and Surgeons. 1000 Tenth Avenue, Suite 3A-02, New York, USA

fDepartment Of Rheumatology, Rush University Medical Center, Chicago, USA

${ }^{g}$ Corresponding author: Girish N. Nadkarni, Department of

Nephrology, The Mount Sinai School Of Medicine, One Gustave L.

Levy Place, No:1243, New York, USA

Email: gnadkarni1178@gmail.com
}

doi:http://dx.doi.org/10.4021/jmc850w and may lead to acute adrenal crisis, shock, and death unless it is recognized promptly and treated appropriately [1]. It has been diagnosed with increasing frequency in hospitalized patients with the advent of newer imaging technology [2]. Adrenal hemorrhage may result from trauma, acute stress, anticoagulation, coagulopathy, underlying malignancy or idiopathic disease [3]. Adrenal hemorrhage due to an infectious etiology is a rare, usually bilateral and seen with meningococcal infections [4]. However, unilateral adrenal hemorrhage caused by Mycobacterium tuberculosis and leading to acute adrenal insufficiency is an exceedingly rare phenomenon and has seldom been described. Here we describe a case of unilateral adrenal hemorrhage in a patient with pulmonary tuberculosis presenting with acute abdominal pain.

\section{Case Report}

A 75-year-old male with past medical history of hypertension, dyslipidemia, diabetes mellitus type 2 and depression presented with a one-month history of productive cough with white sputum. On further inquiry, he reported an unintentional weight loss of 25 pounds in the past 4 weeks and night sweats. Before his presentation he was treated with azithromycin for a presumed community-acquired pneumonia without any improvement in his symptoms. He had recently returned from a 5-month trip to the Dominican Republic. On presentation, he was found to have a blood pressure (BP) of $150 / 90 \mathrm{mmHg}$, pulse of 77 beats/minute, respiratory rate of 18/minute and a temperature of 37 degrees Celsius. Physical examination revealed coarse breath sounds over the right middle and lower zones with the left lung clear to auscultation. A chest Roentgenogram was done which revealed multiple thick-walled, cavitary lesions in right middle and lower lobes with air-fluid levels. Considering these results, an urgent Computerized Tomography (CT) showed multiple irregular thick-walled, cavitary lesions in right middle and lower lobes with adjacent "tree in bud" opacities.

Patient was placed on airborne precautions and the next day of hospitalization sputum staining revealed Acid Fast Bacilli (AFB). A diagnosis of pulmonary tuberculosis was 


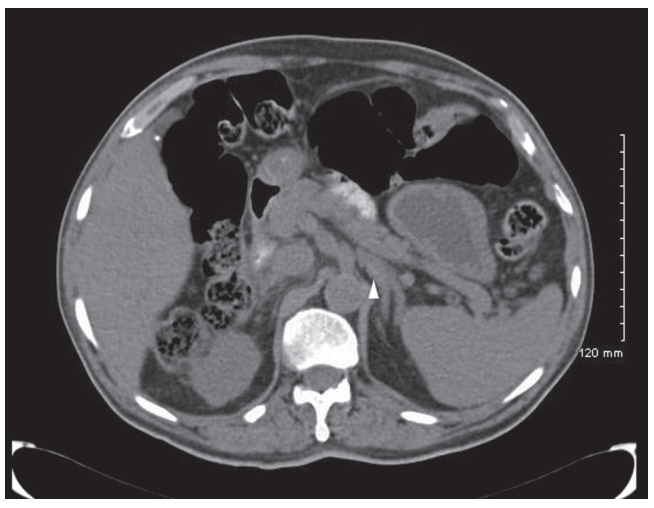

Figure 1. The white arrowhead demonstrates the area of hemorrhage in the left adrenal gland.

made and antitubercular therapy initiated with a 4-drug regimen of ethambutol, pyrazinamide, rifampin and isoniazid. Sputum samples for AFB were monitored every week in order to document mycobacterium tuberculosis clearance. During the 9th day of hospitalization he developed acute flank pain on the right side that was 10/10 in intensity, radiating to his groin, stabbing in nature and not aggravated or alleviated by anything.

Considering these concerning findings, an emergent abdominal CT scan was performed which revealed left side adrenal enlargement with hemorrhagic changes (Fig. 1). He also developed hyponatremia with a sodium of $128 \mathrm{mEQ} / \mathrm{L}$ (131 - $143 \mathrm{mEQ} / \mathrm{L})$; hyperkalemia with serum potassium of $5.5 \mathrm{mg} / \mathrm{dL}(3.5-5.1 \mathrm{meQ} / \mathrm{L})$ and hypoglycemia with serum glucose of $60 \mathrm{mg} / \mathrm{dL}$ with a significant decrease in his insulin requirements. His blood pressure that was usually in the hypertensive range suddenly decreased to $100 / 60 \mathrm{mmHg}$. The right-sided adrenal gland was normal on the CT scan. Laboratory findings revealed an elevated adrenocorticotropic Hormone (ACTH) level at $110 \mathrm{pg} / \mathrm{mL}(20-80 \mathrm{pg} / \mathrm{L})$ and decreased aldosterone levels at $<1.6 \mathrm{ng} / \mathrm{dL}(19-34 \mathrm{ng} / \mathrm{dL})$ which were consistent with acute adrenal insufficiency. The morning serum cortisol level was low to $3 \mathrm{mcg} / \mathrm{dL}$ (6 - 23 $\mathrm{mcg} / \mathrm{dL}$ ). A cosyntropin test was positive with a rise of $<$ $25 \%$ in the serum cortisol level at 90 minutes from a baseline of $4.1 \mathrm{mcg} / \mathrm{dL}$.

An endocrinology consultation was requested and considering a presumptive diagnosis of acute adrenal insufficiency he was treated with Fludrocortisone at a dose of 0.05 $\mathrm{mg}$ every twenty-four hours and Hydrocortisone at a dose of $100 \mathrm{mg}$ every 8 hours. After the treatment his blood pressure stabilized and serum glucose and potassium levels normalized after seven days. The corticosteroids were tapered off over 14 days and the patient continued to maintain his blood pressure. His sodium and potassium levels normalized and his blood glucose came back to his baseline. His ACTH and aldosterone levels normalized indicating recovery in adre- nal function. He continued to receive antitubercular therapy for three months as an inpatient with significant clinical improvement in his cough and sputum clearance of AFB. After three consecutive sputum samples were negative for AFB he was discharged with isoniazid and rifampin for 4 more months. He continued to be monitored on an outpatient basis without any evidence of adrenal insufficiency or recurrence of pulmonary or extra pulmonary tuberculosis.

\section{Discussion}

In the 1900s, tuberculosis was one of the leading causes of death in the United States. However, since then the incidence of tuberculosis has steadily declined due to improvement in living conditions with the incidence reaching the lowest in 2005. However tuberculosis continues to be a significant problem in certain demographic groups including immigrants. The tuberculosis rate in foreign-born persons in the United States was 8.7 times that of U.S. -born persons [5]. Tuberculosis has the potential to affect each organ system of the body. Adrenal tuberculosis usually presents with adrenal insufficiency and is the cause of around $20 \%$ of cases worldwide. Other causes include autoimmune, other infectious diseases primarily meningococcal meningitis, replacement by metastatic cancer or lymphoma, adrenal hemorrhage or infarction, or drugs [6,7]. Adrenal hemorrhage is usually rarer than adrenal insufficiency. Though it can be seen with infectious diseases such as meningococcal disease and malaria, it usually has a more dramatic presentation and occurs bilaterally $[1,4]$.

Unilateral adrenal hemorrhage is uncommon in infectious disease and is seen with blunt trauma, metastatic tumors, coagulation disorders and rarely with long-term nonsteroidal anti-inflammatory drugs usage [3]. There are only a few case reports describing acute adrenal insufficiency caused by unilateral adrenal hemorrhage and its association with pulmonary tuberculosis has been very rarely described [8]. There are very limited data about its presentation, course and outcomes. Fortunately, in our patient's case a high index of suspicion detected the hemorrhage at the initial presentation and timely steps were taken in order to treat the adrenal insufficiency that followed. This timely diagnosis and treatment might have contributed to our patient's excellent outcome.

\section{Conclusions}

Through this case report, we wish to emphasize that a high index of suspicion should be maintained with non-specific abdominal symptoms that present with pulmonary tuberculosis. If acute adrenal insufficiency is not discovered and treated early it could potentially lead to life threatening consequences. 


\section{References}

1. Rao RH. Bilateral massive adrenal hemorrhage. Med Clin North Am. 1995;79(1):107-129.

2. You JS, Chung SP, Park YS, Chung HS, Lee HS, Yu JS. Isolated adrenal hemorrhage after minor blunt trauma. Am J Emerg Med. 2007;25(8):984 e985-986.

3. Simon DR, Palese MA. Clinical update on the management of adrenal hemorrhage. Curr Urol Rep. 2009;10(1):78-83.

4. Manchanda V, Gupta S, Bhalla P. Meningococcal disease: history, epidemiology, pathogenesis, clinical manifestations, diagnosis, antimicrobial susceptibility and prevention. Indian J Med Microbiol. 2006;24(1):7-19.

5. www.cdc.gov [Accessed 28th February, 2012].

6. Irvine WJ, Barnes EW. Adrenocortical insufficiency. Clinics in Endocrinology and Metabolism. 1972;1:549594.

7. Kasperlik-Zaluska AA, Migdalska B, Czarnocka B, Drac-Kaniewska J, Niegowska E, Czech W. Association of Addison's disease with autoimmune disorders--a long-term observation of 180 patients. Postgrad Med J. 1991;67(793):984-987.

8. Polsky EG, Polsky MS, Golden DA. Unilateral adrenal hemorrhage in a patient with Addison's disease. J Urol. 2001;165(6 Pt 1):1982-1983. 